The Astrophysical Journal, 238:502-509, 1980 June 1

(C) 1980. The American Astronomical Society. All rights reserved. Printed in U.S.A.

\title{
RECOMBINATION SPECTRUM AND REDDENING IN NGC 1068
}

\author{
G. Neugebauer, ${ }^{1}$ D. Morton,${ }^{2}$ J. B. Oke ${ }^{1,3}$ E. E. Becklin, ${ }^{4}$ E. Daltabuit,${ }^{5}$ K. Matthews, ${ }^{1}$ S. E. Persson, ${ }^{6}$ \\ A. M. Smith, ${ }^{7}$ B. T. Soifer, ${ }^{1}$ S. Torres-Peimbert, ${ }^{5}$ and C. G. Wynn-Williams ${ }^{4}$ \\ Received 1979 November 7; accepted 1979 December 11
}

\begin{abstract}
Measurements of the emission-line intensities of NGC 1068 have been made over the wavelength range extending from $\lambda_{\text {rest }}=1216 \AA$ to $1.875 \mu \mathrm{m}$. The data, plus other available emission-line data, can be explained in terms of a simple model where the emission lines are formed in an $\mathrm{H}$ II region, and the line ratios are consistent with those predicted by standard radiative recombination theory and reddening corresponding to $E_{B-V}=0.4 \mathrm{mag}$. The continuum flux is seen to consist of a galaxy component plus a nonstellar component which dominates the observed flux in the ultraviolet. The observed ultraviolet continuum does not show an absorption dip caused by the interstellar $2200 \AA$ feature nor does it contain enough energy to power the observed infrared flux.
\end{abstract}

Subject headings: galaxies: individual — galaxies: nuclei — galaxies: Seyfert

\section{INTRODUCTION}

A major problem in the understanding of the nuclei of active galaxies and quasars is that of the hydrogen recombination-line ratios and their relation to extinction within the Galaxy or in the active galaxy itself. It has become clear from the work of Baldwin (1977); Davidsen, Hartig, and Fastie (1977); Hyland, Becklin, and Neugebauer (1978); Boggess et al. (1978); Boksenberg et al. (1978); Puetter, Smith, and Willner (1979); Oke and Zimmerman (1979), and Soifer et al. (1979) that the $\mathrm{H}$ I line ratios, in particular $\mathrm{L} \alpha / \mathrm{H} \alpha$, are anomalous in quasars and some type 1 Seyfert galaxies. It is presently not known whether the observed anomalous line ratios are characteristic of the intrinsic line ratios or a result of dust extinction.

It is important to extend observations of line intensities to as wide a range of active galaxies as possible to determine how ubiquitous the anomalous line ratios are among active nuclei. The Seyfert galaxy NGC 1068, which is archetypical of type 2 Seyfert galaxies, is particularly well suited for a study of this problem since it is sufficiently bright for hydrogen lines to be measured from the ultraviolet (Boksenberg et al. 1978) to the infrared (Thompson, Lebofsky, and Rieke 1978) as well as in the visible (Wampler 1971; Shields and Oke 1975 and references therein). As a type 2

\footnotetext{
${ }^{1}$ Division of Physics, Mathematics and Astronomy, California Institute of Technology.

${ }^{2}$ Anglo-Australian Observatory.

${ }^{3}$ Palomar Observatory, California Institute of Technology.

${ }^{4}$ Institute for Astronomy, University of Hawaii.

5 Instituto de Astronomica, Universidad Nacional Autonoma de Mexico.

${ }^{6}$ Mount Wilson and Las Campanas Observatories, Carnegie Institution of Washington.

${ }^{7}$ NASA Goddard Space Flight Center.
}

Seyfert galaxy it differs from type 1 Seyfert galaxies and quasars in that there is apparently no high-density region $\left(N_{e} \geq 10^{9} \mathrm{~cm}^{-3}\right)$ where broad lines are produced. The line ratios observed in the nucleus of this object may therefore test whether anomalous ratios are associated with the high-density region as suggested by Ferland et al. (1979).

In this paper we present ultraviolet, visual, and infrared observations of emission lines and the continuum in NGC 1068; the observations cover the wavelength region from $L \alpha$ to $P \alpha$. We restrict the study to those lines from which an estimate of the extinction can be made and will try to determine whether or not the data are consistent with simple radiative recombination theory along with standard interstellar reddening. We also discuss the ultraviolet continuum and the energetics which pertain to NGC 1068.

\section{OBSERVATIONS}

Ultraviolet data were obtained with the International Ultraviolet Explorer (IUE) satellite (Boggess et al. 1978); the available spectra are listed in Table 1. The short wavelength cameras (SWP and SWR) cover the spectral range from 1150 to $2000 \AA$, while the long wavelength camera (LWR) goes from 1900 to $3200 \AA$. The $10^{\prime \prime} \times 20^{\prime \prime}$ entrance diaphragm is a $10^{\prime \prime}$ wide slot with $10^{\prime \prime}$ diameter semicircular ends; the area is $179 \operatorname{arcsec}^{2}$. The smaller diaphragm is a $3^{\prime \prime}$ diameter circle.

The $I U E$ spectra used in this paper were reduced by using the photometric transformation established in 1979 July 7 ; they were calibrated absolutely using the mean relations of Bohlin et al. (1979). These calibrations are quite different from the preliminary ones used by Boksenberg et al. (1978). A sample spectrum, 
TABLE 1

IUE SPECTRA OF NGC 1068

\begin{tabular}{|c|c|c|c|c|c|}
\hline Date & Camera $^{\mathrm{a}}$ & No. & $\underset{\left({ }^{\prime \prime}\right)}{\text { Diaphragm }}$ & $\begin{array}{l}\text { Exp. Time } \\
\text { (min) }\end{array}$ & Observer \\
\hline 1978 Feb. 22. & SWR & $1049^{b}$ & $10 \times 20$ & 180 & Commissioning team \\
\hline 1978 June 14 & LWR & 1666 & $10 \times 20$ & 40 & Morton \\
\hline 1978 June 29 & SWP & 1881 & $10 \times 20$ & 20 & Smith \\
\hline 1978 July $1 \ldots$ & SWP & 1898 & $10 \times 20$ & 50 & Daltabuit \\
\hline 1978 July 4 . . & LWR & 1778 & 3 & 80 & Daltabuit \\
\hline$\ldots \ldots \ldots \ldots \ldots$ & SWP & 1921 & 3 & 120 & Daltabuit \\
\hline 1979 August 26 . & SWP & 6285 & $10 \times 20$ & 50 & Morton \\
\hline 1979 August 28 & SWP & 6311 & 3 & 80 & Morton \\
\hline
\end{tabular}

${ }^{a}$ SWR $=$ short wavelength redundant camera; SWP $=$ short wavelength prime camera LWR $=$ long wavelength redundant camera.

${ }^{\mathrm{b}}$ Boksenberg et al. (1978).

absolutely calibrated, taken with the short wavelength camera and the $10^{\prime \prime} \times 20^{\prime \prime}$ diaphragm is shown in Figure 1. The statistical uncertainties in the data range from 5 to $10 \%$, while Bohlin et al. estimate that the uncertainty in the absolute calibration is $10 \%$.

Fluxes in the spectral region from 3200 to $10000 \AA$ were obtained 1978 November 1 with the multichannel spectrometer on the Hale $5 \mathrm{~m}$ telescope. A 10" diameter diaphragm was used and the band passes were $40 \AA$ below $5700 \AA$ and $80 \AA$ above that wavelength. The fluxes are based on the mean absolute calibration of $\alpha$ Lyrae adopted by Hayes and Latham (1975), but smoothed by 0.01 to $0.02 \mathrm{mag}$ to agree with model atmosphere fluxes. The new multichannel observations agree well in absolute flux and relative energy distribution with similar observations made in 1971 and 1972 by Shields and Oke (1975).

On 1978 November 18 several spectra of NGC 1068 were obtained with the cooled SIT digital spectrograph

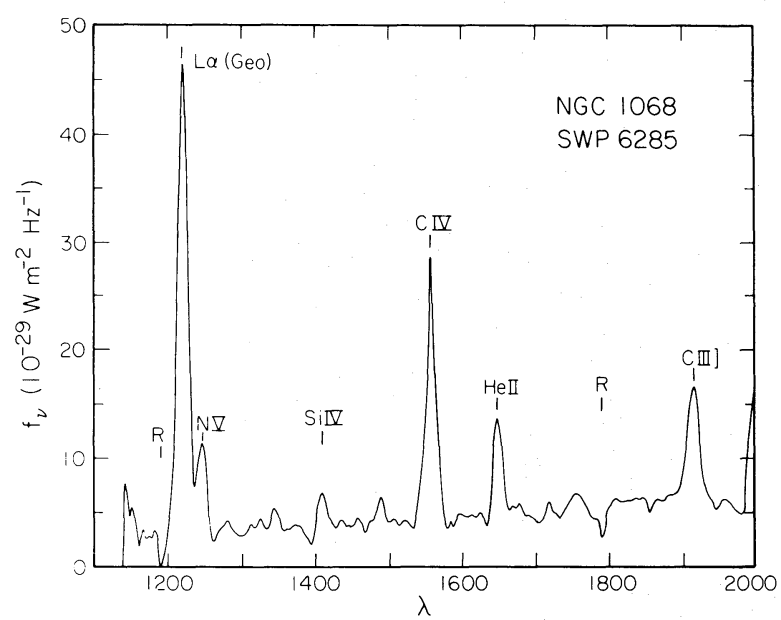

FIG. 1.-An absolutely calibrated IUE short wavelength spectrum of NGC 1068 taken using the $10^{\prime \prime} \times 20^{\prime \prime}$ diaphragm. The spectrum has been smoothed by taking a running mean of 3 pixels ( 1 pixel is $2 \AA$ wide). The line at $1216 \AA$ is almost entirely geocoronal in origin. $\mathrm{R}$ indicates the positions of réseau marks on the detector. on the Hale $5 \mathrm{~m}$ telescope. One pair of spectra was obtained using 12".7 diameter diaphragms, i.e., diaphragms nearly as large in area as the large IUE diaphragm, while the remainder were made with either $8^{\prime \prime}$ diameter diaphragms or a $1^{\prime \prime} \times 60^{\prime \prime}$ slit. The resolution of the slit spectra is about $6 \AA$. The observations made with circular diaphragms have lower resolution but are calibrated absolutely; the fluxes agree with those inferred from the multichannel observations described above. In addition, SIT spectra using the $1^{\prime \prime} \times 60^{\prime \prime}$ slit were obtained on the nucleus and 2".5 and $10^{\prime \prime}$ north of the nucleus on 1975 October 3 .

Infrared observations made to obtain the intensity of $\mathrm{P} \alpha$ at $1.88 \mu \mathrm{m}$ were carried out at the $\mathrm{f} / 35$ Cassegrain focus of the $2.2 \mathrm{~m}$ telescope at the Mauna Kea Observatory in Hawaii. An Ebert-Fastie spectrometer (described by McCammon, Münch, and Neugebauer 1967) was used with a $600 \mathrm{~g} \mathrm{~mm}^{-1}$ grating in first order and an InSb photovoltaic detector. A cooled circular variable filterwheel with a resolution $\Delta \lambda / \lambda$ $=0.013$ was used to reduce the thermal background and define the appropriate grating order. The spectral resolution was $\Delta \lambda \approx 0.006 \mu \mathrm{m}$ full width at halfmaximum transmission for an extended source; this was determined by observing the $\mathbf{B} \gamma$ line at $2.16 \mu \mathrm{m}$ in the extended Orion H II region. A 5"6 circular diaphragm was used for all these observations.

Near the wavelength of the redshifted $\mathrm{P} \alpha$ line, $1.882 \mu \mathrm{m}$, the transmission of the Earth's atmosphere is severely reduced by water vapor lines even at the $4300 \mathrm{~m}$ elevation of Mauna Kea. The transmission thus depends strongly on wavelength, as well as air mass, and is potentially extremely variable in time. Data at each wavelength were obtained by alternating the photometric measurements of NGC 1068 at a given spectrometer and filterwheel wavelength setting with measurements at the same settings of the nearby dF6 star 84 Ceti. When practical, observations were made at wavelengths corresponding to maxima in the Earth's atmospheric transmission.

Observations were obtained on 1978 October 22 and 23. It is of interest to note that on 1978 October 23 the transmission at $1.88 \mu \mathrm{m}$ was approximately four times 

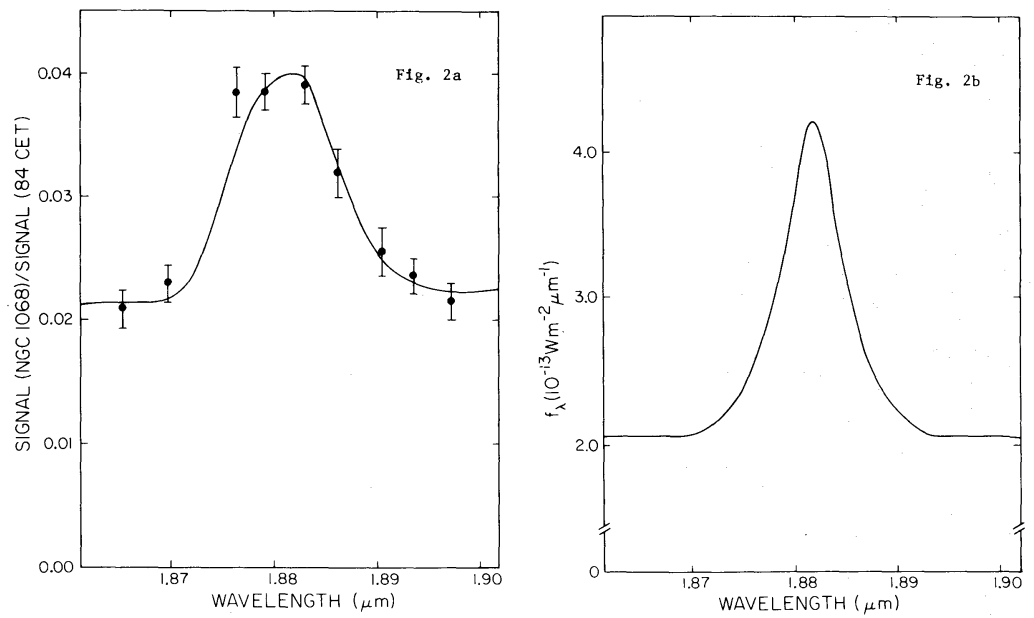

FIG. 2. - The ratio of the infrared flux from NGC 1068 to that of the nearby star 84 Cet is shown in Fig. $2 a$; the spectral resolution is $0.006 \mu$ m. The solid curve in Fig. $2 a$ shows the calculated ratio derived by a convolution of the input spectrum shown in Fig. 2b, the transmission of Earth's atmosphere, and the spectral response of the infrared spectrometer.

that on 1978 October 22; nonetheless, the observed ratios agreed within the statistical uncertainties. The averaged results from both days' observations are shown in Figure $2 a$ as ratios of the flux of NGC 1068 to that of 84 Cet. The measurements are given as ratios rather than as flux densities because the atmospheric transmission varied with wavelength within the spectral bandpass in a way which was not known in detail. The flux densities can thus best be obtained indirectly as described in $\S \mathrm{III} a$. The flux density of $84 \mathrm{Cet}$ at $1.882 \mu \mathrm{m}$ was taken as $9.4 \times 10^{-12} \mathrm{~W} \mathrm{~m}^{-2} \mu \mathrm{m}^{-1}$; this value was obtained from measurements with the spectrometer at $2.1 \mu \mathrm{m}$ of 84 Cet and 35 Pegasi as well as subsequent broad-band $1.65 \mu \mathrm{m}$ and $2.2 \mu \mathrm{m}$ photometry of 84 Cet.

\section{REDUCTION}

\section{a) Emission-Line Intensities}

In order to compare the emission-line intensities measured with different sized diaphragms in the ultraviolet, the visible, and the infrared, some assumption must be made about the region from which the lines arise. In the following discussion the observations were reduced to give line intensities by assuming that

TABLE 2

EMISSION-LiNE EQUIVALENT WIDTHS AND INTENSITIES

\begin{tabular}{lccc}
\hline \hline Identification & $\left(10^{-16} \mathrm{~W} \mathrm{~m} \mathrm{~m}^{-2}\right)$ & $\begin{array}{c}\text { E.W. } \\
(\AA)\end{array}$ & $\begin{array}{c}\text { Diaphragm } \\
(")\end{array}$ \\
\hline $\mathrm{L} \alpha, \lambda 1216 \ldots \ldots$ & $76 \pm 11$ & $123 \pm 17$ & $10 \times 20^{\mathrm{a}}$ \\
$\mathrm{He}$ II, $\lambda 1640 \ldots \ldots$ & $14 \pm 3$ & $28 \pm 5$ & $10 \times 20$ \\
$\mathrm{He}$ II, $\lambda 4686 \ldots \ldots$ & $9 \pm 2$ & $8 \pm 2$ & 12.7 \\
$\mathrm{H} \beta, \lambda 4861 \ldots \ldots$ & $16 \pm 2$ & $15 \pm 2$ & 12.7 \\
$\mathrm{H} \alpha, \lambda 6562 \ldots \ldots$ & $87 \pm 12$ & $82 \pm 11$ & 12.7 \\
$\mathrm{P} \alpha, \lambda 18751 \ldots \ldots$ & $17 \pm 3$ & & 5.6 \\
\hline
\end{tabular}

a Based on equivalent width in 3 " diameter diaphragm. the emission-line region is concentrated into a pointlike source; this assumption implies that for diaphragms larger than a stellar image the derived line intensities are independent of the diaphragm size. Observations which actually give the spatial distribution of line intensities are extremely limited. Measurements of $\mathrm{H} \beta$ in $7^{\prime \prime}, 10^{\prime \prime}$, and 12".7 diameter diaphragms give line intensities of $14.5 \pm 2$ (Shields and Oke 1975), $14 \pm 2$, and $16 \pm 2 \times 10^{-16} \mathrm{~W} \mathrm{~m}^{-2}$, respectively, indicating at most a marginal increase of flux with diaphragm. Similarly, the slit spectra taken off the nucleus of NGC 1068 indicate that the increase in $\mathrm{H} \beta$ line intensity between a $5^{\prime \prime}$ and $15^{\prime \prime}$ diameter diaphragm is less than $20 \%$ of the value inside a $5^{\prime \prime}$ diameter diaphragm centered on the nucleus. In the discussion below an attempt has been made to match diaphragm areas when determining line ratios. The uncertainties in diaphragm dependence are in each case unknown but probably of the same order as the uncertainties in the measurements of the line intensities; no estimate of this effect is included in the stated uncertainties. Line intensities and equivalent widths are given in Table 2 and are discussed individually below.

$L \alpha$-Because of geocoronal contamination, $L \alpha$ in NGC 1068 cannot be measured with the large IUE diaphragm; it can, however, be measured on IUE spectrum SWP 6311 taken with the small diaphragm. Photometrically accurate measurements cannot be made directly with the smaller diaphragms because of the light spillage beyond the diaphragm due to the image quality of the telescope; the effective sampling diameter of the small diaphragm is probably close to $5^{\prime \prime}$. As a result, the line intensity was derived from the continuum flux found in the larger IUE diaphragm and by assuming the equivalent width is the same for both large and small $I U E$ diaphragms. This assumption is valid if both the line and continuum fluxes come from a pointlike region; a model which suggests this is 
appropriate is given below in $\S \mathrm{V}$. In addition, observations show that the equivalent widths of $\lambda 1550$ of C IV and $\lambda 1640$ of He II are the same in both the large and small diaphragms within the uncertainties $( \pm 25 \%)$ of the measurements. The $\lambda 1241$ line of $\mathrm{N} v$ can be separated from $\mathrm{L} \alpha$ quite well.

He II $\lambda 1640$ - This line can be measured with both the large and small $I U E$ diaphragms. Because the line is weak, the uncertainty in each equivalent width is larger than any indicated diaphragm effect. We have therefore used the average equivalent width as measured in SWP 6285 and SWP 6311 combined with the continuum flux measured in the larger diaphragm.

$\mathrm{He}$ II $\lambda 4686, H \beta$, and $H \alpha-\mathrm{New}$ line intensities of $\mathrm{He}$ II $\lambda 4686, \mathrm{H} \beta$, and $\mathrm{H} \alpha$ are based on SIT spectra made with a 12 .7 diameter diaphragm. The $\lambda 4686$ line is blended slightly with other lines, particularly at shorter wavelengths. The $\mathrm{H} \alpha$ line is seriously blended with the two $[\mathrm{N}$ II] lines at $\lambda 6548$ and $\lambda 6583$. A decomposition of the feature was made using the $1^{\prime \prime}$ slit observations which partially resolve the three lines. If all the lines have the same profile, $\mathrm{H} \alpha$ is $0.33 \pm 0.04$ of the whole feature. Eilek et al. (1973), assuming similar profiles, also obtained 0.33 for this ratio while Koski (1978) obtained 0.30 . Observations made on the nucleus and 2 ."5 north of the nucleus in 1975 indicate that this ratio is the same in these two regions. We therefore adopt a ratio of $0.33 \pm 0.04$.

$P \alpha$-The $\mathrm{P} \alpha$ line intensity was found by numerically dividing an assumed line profile for NGC 1068 by a model atmosphere for 84 Cet after each had been convolved by a model for the Earth's atmospheric transmission and the spectrometer bandpass; the resultant ratios were then compared with the observed ratios as shown in Figure $2 a$. The model atmospheric transmission was based on a high-resolution lunar spectrum obtained from Mauna Kea by D. Cruikshank (1978). Computer simulations which incorporate a large range in the absolute level of the transmission show that the measured ratios were insensitive to changes in the transmission. The spectral distribution of $84 \mathrm{Cet}$, including a $20 \%$ absorption dip at $1.875 \mu \mathrm{m}$ due to $\mathrm{P} \alpha$, was obtained from model stellar atmosphere calculations by Peterson (1978).

The spectrum of NGC 1068 was simulated by superposing a $\mathrm{P} \alpha$ emission feature onto a smooth continuum whose slope was derived from broad-band 1.65 and $2.2 \mu \mathrm{m}$ measurements. The shape of the emission feature was calculated by scaling highresolution measurements of the $\mathrm{H} \beta$ line (Sargent and Boksenberg 1979) to the redshifted wavelength of $\mathrm{P} \alpha$ at $1.882 \mu \mathrm{m}$. The best-fit simulation of the observed ratio is included in Figure $2 a$. The spectral distribution assumed for NGC 1068 in this fit is shown in Figure $2 b$; the line intensity taken for the $\mathrm{P} \alpha$ line is $1.7 \pm 0.3$ $\times 10^{-15} \mathrm{~W} \mathrm{~m}^{-2}$. The quoted uncertainty is based on systematic uncertainties, largely in the absolute calibration, and exceeds the statistical uncertainty of the fit.

\section{b) The Continuum Spectrum}

Figure 3 shows the continuum flux of NGC 1068 within a $15^{\prime \prime}$ diameter diaphragm, i.e., one with the same area as the $20^{\prime \prime} \times 10^{\prime \prime} I U E$ diaphragm. The

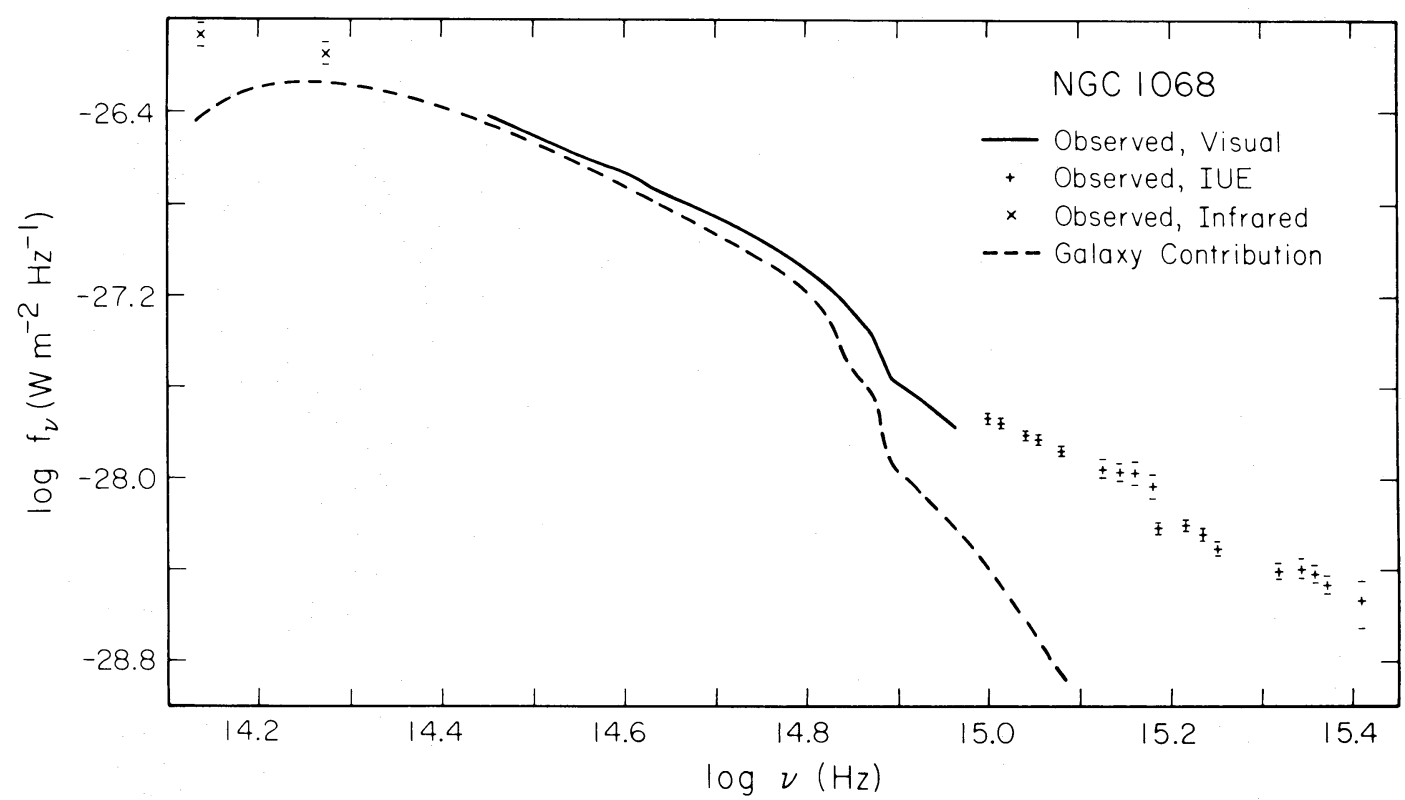

FIG. 3.-The absolute spectral energy distribution of the continuum of NGC 1068 in a $15^{\prime \prime}$ diameter diaphragm from IUE (+), visual ( - ), and infrared $(x)$ data. Standard deviations are shown except in the visual when they are less than 0.02 in $\log f_{v}$. The dashed curve is the estimated contribution of the stellar galaxy light in the observed diaphragm. The continuum of NGC 4472 has been normalized with respect to that of NGC 1068 so as to make the residual between them smooth and relatively flat; see text. 
continuum regions between the various detected emission lines were averaged, using the $20^{\prime \prime} \times 10^{\prime \prime}$ diaphragm low-resolution IUE spectra, to give the ultraviolet fluxes. The corresponding visual continuum was derived from the multichannel observations with a $10^{\prime \prime}$ circular diaphragm. The observed visual fluxes have been increased by 22 to $35 \%$ to correspond to a circular diaphragm of $15^{\prime \prime}$ diameter by using the multiaperture observations of Wampler (1971). The Wampler data were extrapolated to produce corrections below $3650 \AA$. The infrared continuum is represented by the broad-band photometric data at 1.65 and $2.2 \mu \mathrm{m}$ of Neugebauer et al. (1971) for a $15^{\prime \prime}$ diameter diaphragm. There are no significant discontinuities in the continuum fluxes obtained by differing techniques; this suggests that no serious absolute calibration errors are present.

\section{REDDENING}

\section{a) Reddening of Emission Lines}

The line intensities derived in this paper, and others discussed below, can be used to find the interstellar reddening in a number of ways. Values of the extinction, $E_{B-V}$, were derived from a comparison of the measured and theoretical recombination-line ratios and are shown in Table 3. The theoretical line ratios are for case $\mathbf{B}$, radiative recombination, with an electron temperature of $10,000 \mathrm{~K}$ and an electron density of $10^{6} \mathrm{~cm}^{-3}$; the theoretical ratios depend only weakly on these parameters. The reddening was assumed to be due to material, external to the emitting region, which has the extinction curve of Code et al. (1976) extrapolated into the infrared by the van de Hulst curve no. 15 (van de Hulst 1949; Johnson 1968; Becklin et al. 1978). Some implications of this assumption are discussed at the end of this section.
In addition to the line intensities discussed in $\S$ III $a$, additional line intensities measured in NGC 1068 were taken from the literature. For a comparison with the $\mathrm{L} \alpha, \mathrm{P} \alpha$, and $\mathrm{B} \gamma$ lines we have taken $j(\mathrm{H} \beta)=1.45 \pm 2$ $\times 10^{-16} \mathrm{~W} \mathrm{~m} \mathrm{~m}^{-2}$ as measured by Shields and Oke (1975) with a $7^{\prime \prime}$ diameter diaphragm. The B $\gamma$ line intensity is taken from Thompson, Lebofsky, and Rieke (1978) who find $j(\mathrm{~B} \gamma)=1.8 \pm 0.4 \times 10^{-16} \mathrm{~W}$ $\mathrm{m}^{-2}$ in an $8^{\prime \prime}$ diameter diaphragm. Simple recombination theory (Brocklehurst 1971; Giles 1977) gives $j(\mathrm{~B} \gamma) / j(\mathrm{P} \alpha)=0.079$, while the observed ratio is $j(\mathrm{~B} \gamma) / j(\mathrm{P} \alpha)=0.11 \pm 0.04$. Any moderate interstellar extinction does not alter this ratio, and the two infrared measurements are thus in agreement with simple recombination theory. The data on the $\lambda 10320$ and $\lambda 4072$ lines of $[\mathrm{S} \mathrm{II}]$ are taken from Wampler (1968, 1971) and Shields and Oke (1975). Netzer and Davidson (1979) point out that the lines O I $\lambda \lambda 1303$, 8446 offer a good reddening indicator. However, only upper limits on both of these lines can be obtained from the available spectra for NGC 1068, and thus no estimate of the reddening can be derived from the available data.

The individual estimates of $E_{B-V}$ given in Table 3 range from 0.32 to $0.54 \mathrm{mag}$; the weighted mean is $E_{B-V}=0.38 \pm 0.03 \mathrm{mag}$. From a physical point of view the $[\mathrm{S}$ II] and $\mathrm{P} \alpha / \mathrm{H} \beta$ ratios should be the most reliable since both lines come from a common upper level and are thus not as likely to be affected by ambient physical conditions.

Despite the good fit of all the line data to a standard reddening curve, it should be noted that the conditions under which the standard curve was derived, namely extinction by dust grains unassociated with either source or observer, do not necessarily apply in the case of NGC 1068. The location of the dust along the line of sight to NGC 1068 is unknown, but in view of the

TABLE 3

Summary OF Derived EXTINCTIONS FOR NGC $1068^{\mathrm{a}}$

\begin{tabular}{|c|c|c|c|c|}
\hline \multirow[b]{2}{*}{ ION AND LINES USED } & \multicolumn{2}{|c|}{ Ratio } & \multirow[b]{2}{*}{$\Delta A_{\lambda} / E_{B-V}$} & \multirow{2}{*}{$\begin{array}{c}E_{B-V} \\
\text { (mag) }\end{array}$} \\
\hline & Observed & Theory & & \\
\hline H I $(\mathrm{L} \alpha / \mathrm{H} \beta)$. & $5.2 \pm 1.1^{\mathrm{b}}$ & $35^{\mathrm{c}}$ & 6.44 & $0.32 \pm 0.04$ \\
\hline $\mathrm{H}_{\mathrm{I}}(\mathrm{H} \alpha / \mathrm{H} \beta)$ & $5.3 \pm 1.1$ & $2.81^{\mathrm{d}}$ & 1.28 & $0.54 \pm 0.15$ \\
\hline $\mathrm{H}$ I $(\mathrm{P} \alpha / \mathrm{H} \beta)$. & $1.2 \pm 0.3^{\mathrm{b}}$ & $0.337^{\mathrm{d}}$ & 3.34 & $0.41 \pm 0.07$ \\
\hline $\mathrm{H}$ I $(\mathrm{B} \gamma / \mathrm{H} \beta)$. & $0.12 \pm 0.03^{\mathrm{e}}$ & $0.027^{\mathrm{f}}$ & 3.33 & $0.50 \pm 0.08$ \\
\hline He II $(\lambda 1640 / \lambda 4686)$ & $1.6 \pm 0.7$ & $6.81^{\mathrm{g}}$ & 4.19 & $0.38 \pm 0.07$ \\
\hline$\left[\mathrm{S}_{\text {III }}\right](\lambda 10320 / \lambda 4072)$. & $1.9 \pm 0.3^{\mathrm{h}}$ & $0.55^{\mathrm{i}}$ & 3.26 & $0.40 \pm 0.05$ \\
\hline
\end{tabular}

${ }^{a}$ "See text for details. All "theory" refer to simple case B recombination theory for $T_{e}=10,000 \mathrm{~K}, n_{e}$ $=10^{6} \mathrm{~cm}^{-3}$.

${ }^{\mathrm{b}} \mathrm{H} \beta$ from Shields and Oke (1975).

${ }^{c}$ Osterbrock (1974).

${ }^{\mathrm{d}}$ Brocklehurst (1971).

e Thompson et al. (1978).

' Brocklehurst (1971); Giles (1977).

${ }^{\mathrm{g}}$ Seaton (1978).

${ }^{\mathrm{h}}$ Wampler $(1968,1971)$; Shields and Oke (1975).

'Miller (1968). 
galaxy's low inclination and high galactic latitude it is most likely to be physically associated with the central few hundred parsecs of NGC 1068 itself, possibly surrounding the nucleus. In this case the extinction will be confused by two effects. First, the dust may be mixed with the emitting gas, complicating the radiation transfer (Mathis 1970). Second, if the volume occupied by this dust subtends an angle smaller than the telescope beamwidth, then some of the light that has been scattered by dust grains to the side of the source will be able to be seen by the telescope. The significance of this effect depends on the albedo and the angular scattering function of the particles (Jones 1973). If, as is probable, the albedo is wavelengthdependent, then the effective extinction law will be different from that determined under the usual "interstellar" conditions. There is insufficient information both on grain properties and on the geometry of NGC 1068 to enable these corrections to be made; in the following discussion we will therefore assume $E_{B-V}=0.4 \mathrm{mag}$ applies to the line-emitting region.

\section{b) Reddening of the Continuum}

The data of Figure 3 show that the continuum of NGC 1068 can be considered as being made up of several components. In particular, both the multichannel and SIT spectra in the range 14.45 $<\log v(\mathrm{~Hz})<14.85 \quad(4200<\lambda(\AA)<10,000)$ show definite evidence for the presence of galactic stellar radiation in NGC 1068. The large diaphragm observations clearly show the G-band and the $\mathrm{Mg} b$-band, as well as a definite break in the continuum flux at the $\mathrm{H}$ and $\mathrm{K}$ lines of $\mathrm{Ca} \mathrm{II}$; these features are characteristic of nuclear regions of spiral and elliptical galaxies. Furthermore, even in diaphragms as small as 1"35

$\times 1$ ".5, high-dispersion spectra in the $4000 \AA$ spectral region show numerous stellar absorption lines (Richstone and Morton 1975). It will therefore be assumed that there is one component in the continuum of NGC 1068 which can be characterized by the radiation from an elliptical galaxy. A model for this galaxy component using multichannel spectrometer observations of the elliptical galaxy NGC 4472 is shown in Figure 3; the redshifts of both galaxies are similar. A cursory comparison of the distributions of NGC 1068 and NGC 4472 at short wavelengths shows that there is a second continuum component in NGC 1068 which dominates the energy distribution below $4000 \AA$. For conciseness, this additional radiation will be referred to as the "nonstellar component;" it has been discussed by Visvanathan and Oke (1968).

We proceed by first assuming that the galaxy component is like that of NGC 4472 but reddened by normal interstellar extinction and by the same amount as the lines, i.e., $E_{B-V}=0.4 \mathrm{mag}$. To this is added a nonstellar component of arbitrary energy distribution. The maximum galactic contribution occurs if all the continuum at $10000 \AA$ is galactic light. If this is assumed to be the case, the reddened galaxy distri- bution is very faint in the ultraviolet and contributes only $15 \%$ of the total light at the $\mathrm{H}$ and $\mathrm{K}$ lines; virtually no $H$ and $K$ break would be predicted for the composite light, provided, of course, that the nonstellar component does not have an $\mathrm{H}$ and $\mathrm{K}$ break. Since, as seen in Figure 3, a large $\mathrm{H}$ and $\mathrm{K}$ break is observed, we conclude that reddening of the galaxy continuum component by $E_{B-V}=0.4 \mathrm{mag}$ is ruled out.

The possibility that the galaxy continuum is not reddened is attractive because between $4200 \AA$ and $1 \mu \mathrm{m}$ the energy distributions for NGC 1068 and NGC 4472 are almost identical. In Figure 3 an unreddened galaxy contribution is shown which largely eliminates the $\mathrm{H}$ and $\mathrm{K}$ break and the $\mathrm{Mg} b$ and $\mathrm{G}$-band features in the nonstellar component. This decomposition is seen to be satisfactory, and we thus conclude the galaxy component is largely unreddened. Presumably some slight reddening may, however, exist because of dust inferred from the far-infrared emission (Telesco, Harper, and Loewenstein 1976).

The case for or against de-reddening the shortwavelength nonstellar continuum is not clear. The observed IUE spectrum follows a power law of negative slope which resembles that of most objects showing nonthermal emission at visible or ultraviolet wavelengths. There is no evidence for reddening by material having a normal extinction curve such as given by Code et al. (1976). In particular there is no strong absorption at $2200 \AA$ or the sharp decrease at shorter wavelengths; if the extinction corresponded to $E_{B-V}=0.4$ mag a dip by about a factor of 3 at $2200 \AA$ would be expected. The dust in NGC 1068 may well be different from dust in the Galaxy, but for the "dereddened" curve to follow a simple power-law shape the dust must be deficient by a factor of 10 in the constituents that produce the $2200 \AA$ feature and by a factor of 5 in the constituents that produce the rise in extinction at wavelengths $\lambda<1500 \AA$. A qualitatively similar conclusion, based only on short-wavelength ultraviolet data, was drawn by Boksenberg et al. (1978). Further implications of such reddening will be discussed below.

The residual, nongalaxy component is quite uncertain at wavelengths greater than $5000 \AA(\log v[\mathrm{~Hz}]$ $<14.8$ ) because it is derived from a small difference between two large numbers. There is, however, almost certainly an excess at $2.2 \mu \mathrm{m}$ which, from the multiaperture data of Neugebauer et al. (1971), may be associated with a nearly pointlike source in the nucleus of NGC 1068. It is not clear if this radiation component is associated with the nonstellar component seen in the ultraviolet or if it is associated with the compact source seen at 10 and $20 \mu \mathrm{m}$ which has been attributed to thermal reradiation from dust (Becklin et al. 1973; Telesco, Harper, and Loewenstein 1976). It can be noted that if the observed -1.85 power law seen in the ultraviolet data is extrapolated to infrared wavelengths, it would produce a flux density of $0.6 \mathrm{Jy}$ at $2.2 \mu \mathrm{m}$. This is within a factor of 2 of the flux 
estimated for the unresolved central point source of NGC 1068 by Neugebauer et al.

\section{DISCUSSION}

Perhaps the most important aspect of the data presented here is the comparison with the anomalous emission-line intensity ratios found in quasars and type 1 Seyfert galaxies. The data summarized in Table 3 indicate that the line ratios in NGC 1068 can be explained simply by radiative recombination plus extinction which is presumably within the very central region of the galaxy. It is tempting to speculate that the absence of anomalous line ratios in NGC 1068 is a consequence of the lack of a high-density region which produces the broad emission lines and distinguishes NGC 1068, as a type 2 Seyfert galaxy, from type 1 Seyfert galaxies and quasars. In this connection, Ferland et al. (1979) have shown that in 3C 390.3 the anomalous line ratios are in fact associated with the broad line region, while the lines produced in the narrow line region agree with simple recombination models. In addition, Osterbrock (1979) has summarized data to show that in type 1 Seyfert galaxies the broad line component often has anomalous Balmer line ratios. The observations of a complete set of line ratios, such as given for NGC 1068 above, to type 1 Seyfert galaxies and to quasars are necessary to clarify the picture.

It is of interest that the [S II] lines show the same reddening and line widths as the permitted hydrogen and helium lines (Shields and Oke 1975). Whereas the polarization and position angle of the permitted lines are similar to that of the nonstellar continuum, the forbidden lines are unpolarized (Angel et al. 1976). Thus the [S II] lines probably do not come from precisely the same volume as the permitted lines and continuum although they all come from a region no larger than $1^{\prime \prime}$ in diameter.

Consideration of the energetic constraints provided by the present data provides an insight into the nature of NGC 1068. In particular, the ultraviolet nonstellar continuum is a potentially important source of ionizing photons. If the gas in NGC 1068 is photoionized, then the rate of production of hydrogen ionizing continuum photons should exceed the rate of emission of $\mathrm{L} \alpha$ photons. If the $\mathrm{L} \alpha$ is reddened by $E_{B-V}$ $=0.4 \mathrm{mag}$, the intrinsic $\mathrm{L} \alpha$ flux is $2 \times 10^{-13} \mathrm{~W} \mathrm{~m}^{-2}$, corresponding to $1.2 \times 10^{5}$ photons $\mathrm{m}^{-2} \mathrm{~s}^{-1}$. Extrapolation of the observed power-law continuum, however, gives a total flux of only $1.9 \times 10^{4}$ photons $\mathrm{m}^{-2} \mathrm{~s}^{-1}$, a factor of 6 less than the number implied by the reddening-corrected $L \alpha$ flux. The implication of this result is that there must be some additional source of excitation in NGC 1068 if the uncorrected energy distribution pertains.

In addition, the infrared observations of NGC 1068, as summarized by Telesco et al., show a significant emission in the far-infrared. In particular, there is a local inflection of $\sim 10^{-11} \mathrm{~W} \mathrm{~m}^{-2}$ in the energy distri- bution at about $20 \mu \mathrm{m}$ which, from the size measurement at $10 \mu \mathrm{m}$, is presumably associated with the 1 " nuclear emission. The power observed in the ultraviolet continuum at wavelengths longward of $10^{15} \mathrm{~Hz}$ is only about $3 \times 10^{-13} \mathrm{~W} \mathrm{~m}^{-2}$. The observed ultraviolet continuum uncorrected for extinction is therefore inadequate to produce the infrared luminosity of NGC 1068.

One possibility is that the ultraviolet source is reddened by dust which differs significantly from that in the Galaxy in not showing the $2200 \AA$ absorption feature. In this picture, the source of the nonstellar continuum component and the permitted emission lines lies within the central $1^{\prime \prime}$ of NGC 1068. Within or around this region there are probably individual clouds of dust which redden both of these radiation sources. Outside of this region the observed radiation is again dominated by a stellar galactic component which is not reddened significantly by the dust clouds. If the extinction curve of Code et al. (1976) is applied to the observed data with the arbitrary assumption of a smooth extrapolation which excludes the $2200 \AA$ absorption feature and rise at wavelengths $\lambda<1500 \AA$, the resultant flux density is essentially flat with frequency with a value $\sim 1 \times 10^{-27} \mathrm{~W} \mathrm{~m}^{-2} \mathrm{~Hz}^{-1}$. If this flux density is arbitrarily assumed to be cut off at $380 \AA$ (3 R) the ultraviolet flux is $\sim 8 \times 10^{-12} \mathrm{~W} \mathrm{~m}^{-2}$, comparable to the infrared flux of $10 \times 10^{-12} \mathrm{~W} \mathrm{~m}^{-2}$. The number of photons beyond the Lyman continuum is, under the same assumptions, about 5 times that emitted in $L \alpha$.

A second possibility is that the central 1 " region of the nucleus of NGC 1068 contains a number, perhaps 10 , of compact nonthermal sources, rather than only one. Most of these are hidden but one happens to have negligible extinction. Excited by these ultraviolet sources are the ionized regions, which typically show extinction of $E_{B-V} \sim 0.4 \mathrm{mag}$. The dust associated with these regions is heated to produce the infrared emission seen at $10 \mu \mathrm{m}$. Surrounding this region, and therefore not subject to significant extinction, is the galactic stellar component. On this model the observed ultraviolet energy distribution will be dominated by the source with the least extinction, while the other, more heavily obscured sources provide the bulk of the gas ionization and heating. The observed behavior of the linear and circular polarization (Angel et al. 1976) would be produced by scattering off a dust cloud near the nonthermal source rather than by extinction.

Neither of the above explanations is totally satisfactory. The first requires an extinction curve which is grossly different from any previously published. Although variations in the strength of the $2200 \AA$ feature have been reported, in no case is the feature absent to the degree postulated above (Savage and Mathis 1979 and references therein). Furthermore, changes in the strength of the absorption feature are generally accompanied by changes in the overall extinction curve which would throw doubt on the reddening applied to the line-emitting region. The 
second requires a special geometry so that the ultraviolet sources are visible to the ionized gas but are invisible from the Earth. The present data do not let us distinguish between these two alternatives.

Finally, an obvious suggestion is that strong sources are present which emit at ultraviolet or X-ray wavelengths shortward of those observed. This suggestion appears ad hoc and we do not consider it satisfactory, particularly in view of the low X-ray flux density, $\sim 3$ $\times 10^{-31} \mathrm{~W} \mathrm{~m} \mathrm{~m}^{-2} \mathrm{~Hz}$, observed at $0.3 \mathrm{keV}(7$ $\times 10^{16} \mathrm{~Hz}$ ) (Helfand 1979).

\section{CONCLUSIONS}

1) The emission lines in NGC 1068 do not show the anomalous line ratios which have been found in some quasars and type 1 Seyfert galaxies.
2) The extinction to the emission-line region in NGC 1068 typically corresponds to $E_{B-V}=0.4 \mathrm{mag}$.

3) There must be additional sources of ultraviolet emission which are either heavily obscured or the reddening must be considerably different from that observed in the Galaxy.

We thank D. Peterson for providing us with stellar models, D. Cruikshank for giving us the spectrum of the atmosphere above Mauna Kea, W. Sargent and D. Helfand for providing observations prior to publication, and W. Stein for commenting on the text. The work has been supported by NASA grants NGL 05002-207, NSG 5243, NGL 05-002-134, and NSF grant AST77-20516.
Angel, J. R. P., Stockman, H. S., Woolf, N. J., Beaver, E. A., and Martin, P. G. 1976, Ap. J. (Letters), 206, L5.

Baldwin, J. A. 1977, M.N.R.A.S., 178, 67P.

Becklin, E. E., Matthews, K., Neugebauer, G., and Willner, S. P. 1978, Ap. J., 220, 831

Becklin, E. E., Matthews, K., Neugebauer, G., and Wynn-Williams, C. G. 1973, Ap. J. (Letters), 186, L69.

Boggess, A., et al. 1978, Nature, 275, 361

Bohlin, R. C., Holm, A. V., Savage, B. D., Snijders, M. A., and Sparks, W. M. 1979, preprint

Boksenberg, A., et al. 1978, Nature, 275, 404

Brocklehurst, M. 1971, M.N.R.A.S., 153, 471.

Code, A. D., Davis, J., Bless, R. C., and Hanbury-Brown, R. 1976, Ap. J., 203, 417 .

Cruikshank, D. 1978, private communication

Davidsen, A. F., Hartig, G. F., and Fastie, W. G. 1977, Nature, 269, 203.

Eilek, J. A., Auman, J. R., Ulrych, T. J., Walker, G. A. H., and Kuhi, L. V. 1973, Ap. J., 182, 363.

Ferland, G. J., Rees, M. J., Longair, M. S., and Perryman, M. A. C. 1979, M.N.R.A.S., 187, 65P.

Giles, K. 1977, M.N.R.A.S., 180, 57P.

Hayes, D. S., and Latham, D. W. 1975, Ap. J., 197, 593.

Helfand, D. 1979 , private communication.

Hyland, A. R., Becklin, E. E., and Neugebauer, G. 1978, Ap. J. (Letters), 220, L73.

Johnson, H. L. 1968, in Nebulae and Interstellar Matter, ed. B. M Middlehurst and L. H. Aller (Chicago: University of Chicago Press), p. 167

Jones, T. W. 1973, Pub. A.S.P., 85, 811.

Koski, A. T. 1978, Ap. J., 223, 56.
Mathis, J. S. 1970, Ap. J., 159, 263.

McCammon, D., Münch, G., and Neugebauer, G. 1967, Ap. J., 147, 575.

Miller, J. S. 1968, Ap. J., 154, L57.

Netzer, H., and Davidson, K. 1979, M.N.R.A.S., 187, 871.

Neugebauer, G., Garmire, G., Rieke, G. H., and Low, F. J. 1971, Ap J. (Letters), 166, L45.

Oke, J. B., and Zimmerman, B. A. 1979, Ap. J. (Letters), 231, L13.

Osterbrock, D. E. 1974, Astrophysics of Gaseous Nebula (San Francisco: Freeman).

- 1979, A.J., 84, 901

Peterson, D. 1978, private communication.

Puetter, R. C., Smith, H. E., and Willner, S. P. 1979, Ap. J. (Letters), 227, L5.

Richstone, D. O., and Morton, D. C. 1975, Ap. J., 201, 289.

Sargent, W. L. W., and Boksenberg, A. 1979, private communication.

Savage, B. D., and Mathis, J. S. 1979, Ann. Rev. Astr. Ap., 17, 73.

Seaton, M. J. 1978, M.N.R.A.S., 185, 5P.

Shields, G. A., and Oke, J. B. 1975, Ap. J., 97, 5.

Soifer, B. T., Oke, J. B., Matthews, K., and Neugebauer, G. 1979, Ap. J. (Letters), 227, L1.

Telesco, C. M., Harper, D. A., and Loewenstein, R. F. 1976, Ap. J. (Letters), 203, L53.

Thompson, R. I., Lebofsky, M. J., and Rieke, G. H. 1978, Ap. J. (Letters), 22, L49.

Van de Hulst, H. C. 1949, Rech. Astr. Obs. Utrecht, 11, pt. $1,1$.

Visvanathan, N., and Oke, J. B. 1968, Ap. J. (Letters), 152, L165.

Wampler, E. J. 1968, Ap. J. (Letters), 154, L53.

. 1971, Ap.J., 164, 1.

E. E. BeCKLIN and C. G. WynN-Williams: Institute for Astronomy, University of Hawaii, 2680 Woodlawn Drive, Honolulu, Hawaii 96822

E. Daltabuit and S. Torres-Peimbert: Instituto de Astronomica, Universidad Nacional Autonoma de Mexico, Adpo. Postal 70-264, Mexico 20, D.F., Mexico

K. Matthews, G. Neugebauer, and B. T. Solfer: Division of Physics, Mathematics and Astronomy, Downs Lab 320-47, California Institute of Technology, Pasadena, CA 91125

D. Morton: Anglo-Australian Observatory, Box 296, Epping, NSW 2121, Australia

J. B. OKE: Division of Physics, Mathematics and Astronomy, Robinson 105-24, California Institute of Technology, Pasadena, CA 91125

S. E. Persson: Mount Wilson and Las Campanas Observatories, 813 Santa Barbara St., Pasadena, CA 91101

A. M. Smith: NASA Goddard Space Flight Center, Code 681, Greenbelt, MD 20771 